\title{
Combining high-speed planar PIV and motion tracking of a flexible cylinder in cross-flow
}

\author{
D. G. Gundersen ${ }^{1}$, K. T. Christensen ${ }^{1,2}$, G. Blois ${ }^{1}$ \\ ${ }^{1}$ University of Notre Dame, Department of Aerospace and Mechanical Engineering, Notre Dame, USA \\ ${ }^{2}$ Illinois Institute of Technology, Depts. of Mechanical, Materials \& Aerospace Engineering and Civil, \\ Architectural \& Environmental Engineering, Chicago, USA
}

\section{Introduction}

Most modeling studies investigating the flow dynamics in vegetation canopies are limited to rigid models as proxies for vegetation elements. However, most canopies embody some degree of structural flexibility, resulting in aeroelastic mechanisms coupling the motion of the vegetation with the surrounding flow. Studies addressing flexible canopies typically quantify either the flow or the plant motion independently, thus missing the instantaneous coupling between turbulent stresses and structural deformations. Few experiments have been devoted to measuring both quantities simultaneously. Okamoto and Nezu (2009) utilized a combined PIV-PTV technique to capture both flow and canopy motion. However, only the motion of the stem tips was captured, as opposed to the deformation of the entire stem. Py et al. (2006) employed digital image correlation (DIC) to quantify the motion of crop canopies using in-field images. However, the wind itself was not measured across the domain.

The present work presents an experimental technique that can be utilized to study the flow-structure interaction in flexible canopies, and that could be extended to other flexible and/or moving objects. High-speed PIV data of the flow surrounding an idealized canopy element, consisting of a flexible cylinder, together with the corresponding displacement field throughout the cylinder were simultaneously obtained combining fluorescent imaging and refractive index matching (RIM).

\section{Methods}

Laboratory experiments were performed in the Refractive Index-Matched (RIM) flow facility at Notre Dame (Blois et al., 2020). The RIM approach involved using an aqueous solution of sodium iodide (NaI) as the working fluid and solid models whose RI matches that of the fluid, thus obtaining an optical continuum between the solid model and the surrounding fluid. The operating principle of the technique presented herein involved seeding the two phases with different tracers, facilitating image segmentation and interrogation of each effect independently. The working fluid was seeded with silver-coated particles with a 14-micron diameter and a specific gravity of 1.7. The cylinder models were fabricated from a polyurethane rubber with an index of refraction of $n=1.4865$ and a Young's Modulus of $E=2.9 \mathrm{MPa}$. A small volume of orange fluorescent dye was embedded within the polyurethane during the casting process at a concentration of $1 \mu \mathrm{L} / \mathrm{g}$. The casting protocol was refined over multiple iterations to maximize particle dispersion uniformity and to optimize dye-to-solid ratio. The cylinder was mounted on the bottom wall of the test section with its axis oriented in the normal direction. The cylinder had a length of $L=43 \mathrm{~mm}$ and a diameter of $D=4.5 \mathrm{~mm}$. The model was fully immersed in the boundary layer, with a thickness of $\delta_{99}=58.4 \mathrm{~mm}$. The laser sheet was oriented in the streamwise-wall-normal plane intersecting the center of the cylinder. All particles were illuminated by the same laser sheet formed from a $50 \mathrm{~mJ}$, dual-head Nd:YLF high-speed laser. A single high-speed digital camera was available for these tests. The camera captured both the light scattered by the silver-coated particles seeding the fluid flow and the light emitted by the particles seeded within the cylinder. The signal was separated using robust segmentation algorithms. The displacement field at any point in the flow and within the cylinder was obtained by standard cross-correlation schemes. The segmentation scheme was automated, allowing for the very large datasets produced by the high-speed imaging arrangement to be processed. 
(a)

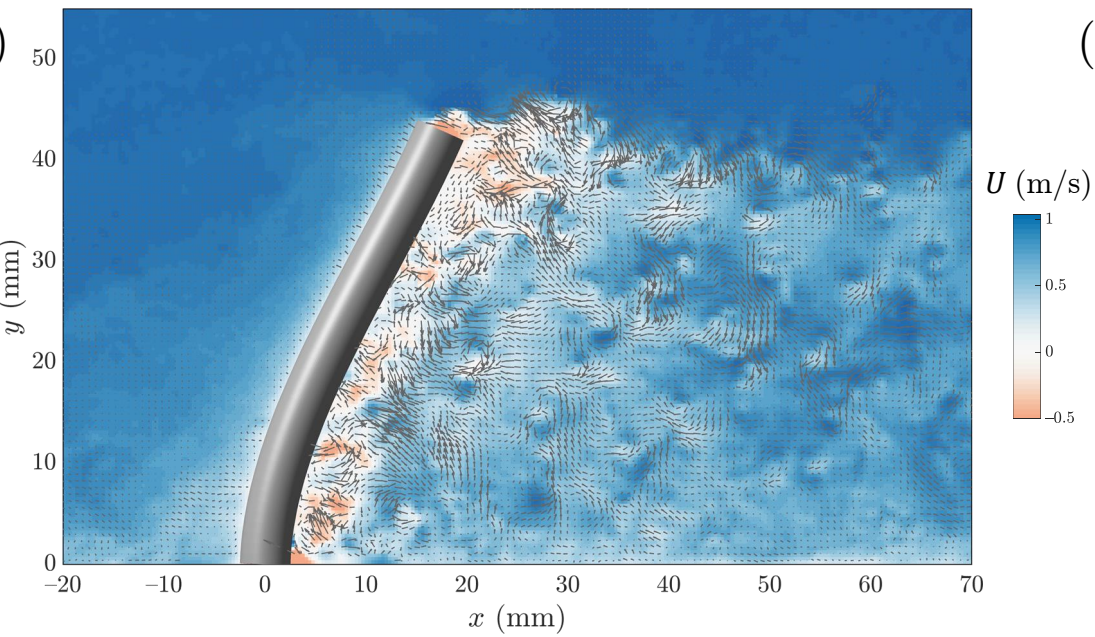

(b)

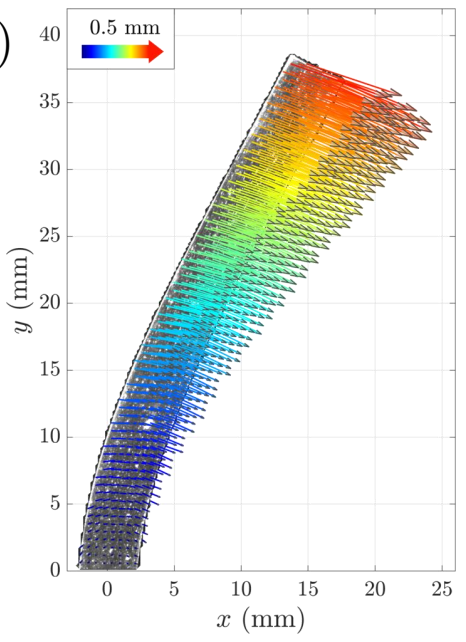

Figure 1: (a) Instantaneous snapshot of the streamwise velocity, $U$, with vectors displaying the fluctuating velocities. The cylinder location is shown in gray. (b) Instantaneous displacement vectors relative to the previous image. For better visualization, the vector magnitudes are exaggerated by a factor of 10 , and a reference vector of $0.5 \mathrm{~mm}$ is shown in the top left.

\section{Results}

Time-resolved planar PIV data was acquired with a sample frequency of 640 image pairs per second and a sample size of 2734 image pairs. The flow data were processed with an iterative multi-pass interrogation method, resulting in a vector grid spacing of $0.33 \mathrm{~mm}$. A representative example of the instantaneous distribution of streamwise velocity, $U$, around the cylinder model subjected to an oncoming turbulent boundary layer is shown in Fig. 11(a). The results shows that, despite a slight decrease in illumination energy in the shadow region underneath the cylinder, the data quality is relatively uniform throughout the field of view. Fig. 11(b) shows the cylinder displacement map at a given snapshot. The vectors are oriented perpendicular to the model centerline and the vector magnitudes increase with distance from the base. These observations are qualitatively consistent with the expected displacement distribution of a cantilever beam that is fixed to the wall and subjected to an oncoming turbulent boundary layer. This experimental technique allowed for the accurate calculation of quantities such as power spectra of the cylinder vibrations and the vortex shedding frequency. In addition, it also facilitated temporal correlations between the cylinder and flow dynamics.

\section{Conclusions}

The preliminary results presented herein demonstrate a technique to successfully embed fluorescent particles in a transparent flexible vegetation model. The utilization of this model in a RIM environment allows one to quantify the motion and deformation of the illuminated object subjected to a turbulent flow. High-speed PIV data allowed for the observation of the dynamic link between a deformable object and the surrounding flow, thus giving the ability to probe the fluid-structure coupling. The experimental method could be utilized to aid in the study of other aeroelastic flow-structure interactions.

\section{References}

Blois G, Bristow NR, Kim T, Best JL, and Christensen KT (2020) Novel environment enables piv measurements of turbulent flow around and within complex topographies. Journal of Hydraulic Engineering 146:04020033

Okamoto TA and Nezu I (2009) Turbulence structure and "monami" phenomena in flexible vegetated openchannel flows. J Hydraul Res 47:798-810

Py C, De Langre E, and Moulia B (2006) A frequency lock-in mechanism in the interaction between wind and crop canopies. J Fluid Mech 568:425-449 